\title{
Validation of the French Version of the Fear of COVID-19 Scale and its Associations with Depression, Anxiety and Differential Emotions
}

\section{Mélody Mailliez}

ISAE-SUPAERO (Institut supérieur de l'aéronautique et de l'espace), Univ. Toulouse (Université Fédérale de Toulouse Midi-Pyrénées), F-31000 Toulouse, France. https://orcid.org/0000-0003-2425-0752

\section{Mark D. Griffiths}

International Gaming Research Unit, Psychology Department, Nottingham Trent University, Nottingham NGI 4FQ, UK. https://orcid.org/0000-0001-8880-6524

Arnaud Carre ( $\nabla$ arnaud.carre@univ-smb.fr)

Univ. Savoie Mont Blanc, Univ. Grenoble Alpes, LIP/PC2S, F-73000, Chambéry, France. https://orcid.org/0000-0001-9914-5565

\section{Research Article}

Keywords: COVID-19, Fear, Fear of COVID-19 Scale, Mental Health, Scale development, French validation

Posted Date: July 22nd, 2020

DOI: https://doi.org/10.21203/rs.3.rs-46616/v1

License: (c) (1) This work is licensed under a Creative Commons Attribution 4.0 International License. Read Full License

Version of Record: A version of this preprint was published at International Journal of Mental Health and Addiction on July 23rd, 2021. See the published version at https://doi.org/10.1007/s11469-021-00499-x. 


\section{Abstract}

The COVID-19 crisis represents a global health concern, including mental health. Regarding the necessity to assess anxiety related to COVID-19, a scale was developed (Ahorsu et al., 2020). The goal of the present study was to validate of the Fear of COVID-19 Scale (FCV-19S) in French (official language in 29 countries), and investigate its relationship with indicators of affective disturbances.

The study was performed on 316 participants. The FCV-19S underwent a double back translation procedure. Its psychometric properties were evaluated.

The French FCV-19S demonstrated a stable unidimensional structure with robust psychometric properties (strong internal consistency, good convergent and divergent validity, and good test-retest validity).

With its robust psychometric properties in assessing the unidimensional construct of the fear of COVID19 , this scale will help to provide a better understanding of the relationship between emotions and psychological or psychiatric disorders during the pandemics in French-speaking countries.

\section{Background}

During the last quarter of 2019 and in the beginning of 2020, a new and highly contagious disease coronavirus disease-2019 (COVID-19) - spread worldwide. To date (July 2020), this pandemic has infected over $10,000,000$ people and caused over 500,000 deaths among more vulnerable individuals (Worldometer, 2020). This crisis caused by COVID-19 is a virologic and infectious problem. If a large part of the contamination leads to asymptomatic or paucisymptomatic cases, some infected individuals will experience serious complications. These complications can be serious and can cause respiratory, inflammatory, and neurological problems (Wu et al., 2020).

The spread of COVID-19 also has an emotional dimension and could also lead to a 'pandemic fear'. For many years, epidemics and their associated protective measures such as lockdowns and quarantining, have been known for their effects on mental health (Brooks et al., 2020; Rogers et al., 2020). One of the major effects is the increase in stress levels and negative affects such as fear (Centers for Disease Control and Prevention, 2020). In addition, ambiguous or even false information about factors related to the COVID-19 (e.g., transmission, incubation period, infection, and mortality rates) can lead to insecurity and fear in the population (Ornell et al., 2020). In such a context, individuals naturally began to be worried about COVID-19, especially of being in contact with individuals who are possibly affected by the COVID19 (Lin, 2020).

All of these negative emotional reactions can have a negative effect on mental health that could evolve into severe disorders including depression, anxiety, paranoia, psychotic episodes, and (in extreme situations) suicide (Griffiths \& Mamun, 2020; Mamun \& Griffiths, 2020). Such abnormal affective states 
can result in irrational processing of information related to the pandemic (Gunnell et al., 2020). Mental health conditions also tend to last longer (e.g., possibly six months after the end of the disease) and are associated with a greater prevalence (Brooks et al., 2020; Ornell et al., 2020; Shultz et al., 2016). It has also been suggested that the 'second wave' of COVID-19 will be more about mental health (World Health Organization [WHO], 2020). Indeed, beyond the re-emergence of the virus in a population a few months apart, public health systems could be faced with the delayed consequences of the pandemic on mental health (Carbone, 2020).

Recently, a self-report psychometric tool - the Fear of COVID-19 Scale (FCV-19S) - was developed to assess the level of fear of COVID-19 among individuals in the general population (Ahorsu et al., 2020). The tool was developed based on the review and assessment of 30 other 'fear scales' (e.g., fear of cancer, fear of crime, fear of missing out in social media use). The most relevant and salient items were selected resulting in 28 relevant items. These items were them reduced to 17 after examination by experts in general health, mental health and virology. Supplementary steps led to the development of a 10-item scale that was then tested and validated among 717 Iranian participants in the Persian language. After testing the psychometric properties of the scale (e.g. exploratory factor analysis, correlation analyses), only seven of the 10 items were retained in the final scale. Since then, it has been translated and validated into many languages including English, Spanish, Russian, Chinese, Japanese, Bangla, Vietnamese, Arabic, Hebrew, Italian, Greek, and Turkish (Mamun et al., 2020). However, it has not been translated and validated in French, the official language in 29 countries worldwide. Considering the role of fear in mental health and its high prevalence in a pandemic, a French version of the scale would help in the understanding of psychiatric and psychological consequences of the COVID-19 in French-speaking countries. As well as translating and validating the FCV-19S in French, the study also assessed its relationship with other markers of affective functioning, such as feeling of differential emotions as well as depression and anxiety.

\section{Methods}

\section{Participants and procedure}

316 French participants ( 271 women) took part in an online survey during the lockdown to inhibit the spread of COVID-19 in France. They were aged between 16 and 77 years ( $M=35.55$ years, $S D=14.27)$. All were French native speakers or totally fluent. Participants were volunteers and recruited through online advertisement on social media (e.g., Facebook and Twitter) and institutional mailing lists. They completed and signed a consent form prior to data collection.

\section{Ethical standards}


All procedures complied with the ethical standards of the relevant national and institutional committees on human experimentation and with the Helsinki Declaration of 1975, as revised in 2008. Additionally, the study was approved by the ethical committee of the University (CER-flash-2020-283).

\section{Measures}

Demographic questions were asked including gender, age, education, occupation, and history of their psychological problems (e.g., depression, anxious disorders), and consumption of psychoactive substances (e.g., nicotine, alcohol, recreational and illicit drugs). The survey also included several psychometric instruments that assessed affective components. Descriptive statistics and reliability coefficients are shown in Table 1.

The Fear of COVID-19 Scale (FCV-19S). The seven-item French version of the Fear of COVID-19 Scale (Ahorsu et al., 2020) was used to assess the extent of fear of COVID-19. Participants provide ratings on a five-point Likert type scale ranging from 1 (Strongly disagree) to 7 (Strongly agree). The higher the score, the greater the fear of COVID-19. Following general guidelines for cross-cultural adaptation (Guillemin et al., 1993), the original items of the FCV- $19 S$ were translated from English to French by two French native speakers. A committee comprising two experts established a preliminary version of the French FCV-19S based on these translations. This preliminary version was then translated from French to English (i.e., back-translation) by an English native speaker. Finally, the committee revised the preliminary version of the FCV-19S based on the translations and back translations (see APPENDIX A for the items). Example items include "I am most afraid of coronavirus-19" and "It makes me uncomfortable to think about the coronavirus-19".

Differential Emotions Scale IV (DES-IV). The DES-IV is a 36-item scale that assesses individuals' trait emotions (i.e., individuals' frequent experiences of discrete emotions in their daily life) on 12 dimensions developed by Izard et al. (1993; French version: Ricard-St-Aubin et al., 2010). Each of the 12 dimensions is assessed by three items. Participants are asked to rate how often they feel the emotions in their daily life on a five-point Likert type scale from 1 (Rarely or never) to 5 (Very often). The higher the score, the greater individuals feel the associated emotions. Positive emotions refer to joy, seeking-interest, and surprise whereas negative emotions refer to anger, disgust, contempt, fear, guilt, shame, shyness, sadness, and self-hostility1. Example items include "In your everyday life, how often do you feel yelling at someone or banging something?" (anger) and "In your everyday life, how often do you feel satisfied about something?" (joy). The scale had acceptable reliability in the present study (Table 1).

The Hospital Anxiety and Depression Scale (HADS). The HADS (Zigmond \& Snaith, 1983; French version: Ravazi et al., 1989) was used to assess the level of depression among participants. Participants provide ratings on a four-point Likert type scale ranging from 0 to 3 . The HADS contains two sub-scales of seven items, one for anxiety and one for depression. Example items include "I feel tense or 'wound up" (anxiety) 
and "I still enjoy the things I used to enjoy" (depression). The HADS is scored by summing the rating for the 14 items yielding a total score that can range from 0 to 42 , and by summing the rating for the seven items of each subscale. The subscales yield separate scores for anxiety and depression that range from 0 to 21 . The scale had acceptable reliability in the present study (Table 1).

Spielberger's State-Trait Anxiety Inventory (STAI). The STAI state (STAI-A) and trait (STAI-B) assesses two dimensions of anxiety (Spielberger et al., 1983; French version: Bruchon- Schweitzer and Paulhan, 1993). The STAI-A and the STAI-B comprises a 20-item scale. Example items include "I feel nervous and restless" and "I feel like a failure". Participants provide ratings on a four-point Likert type scale. Assessment of state anxiety asks participants to describe how they feel "right now, at this moment". In contrast, trait anxiety asks participants to describe how they feel "in general". Each scale is scored by summing the rating for the 20 items to give a score that can range from 20 to 80 . The scales had acceptable reliability in the present study (Table 1).

Perceived Vulnerability to Disease Scale (PVDS). The PVDS (Duncan et al., 2009) is a 15- items scale that assess self-perception of vulnerability to infectious disease specifically perceived infectability (seven-item subscale) and germ aversion (eight-item subscale). Participants respond to each item on a seven-point scale Likert type scale from 1 (strongly disagree) to 7 (strongly agree) with approximately half of the items reverse-scored. Example items include "In general, I am very susceptible to colds, flu and other infectious diseases" (perceived infectability) and "I prefer to wash my hands pretty soon after shaking someone' hand" (germ aversion). A higher score indicates greater perceived infectability, germ aversion and perceived vulnerability to disease (as a whole). The scale was translated into French using the same procedure (outlined above) as that of the French FCV-19S. The French PVDS was used for the first time in the present study, therefore, a confirmatory factor analysis (CFA) was carried out. Results of the CFA $(\chi 2[71]=56.73$; relative $\chi 2=0.79, \mathrm{RMSEA}=.00[95 \% \mathrm{Cl} .00, .01], \mathrm{CFI}=1.00, \mathrm{TLI}=1.02, \mathrm{SRMR}=.04)$ indicated the scale as valid and reliable for assessing perceived infectability and germ aversion. Moreover, analysis $(\chi 2[70]=78.58$; relative $\chi 2=1.12, \mathrm{RMSEA}=.02[95 \% \mathrm{Cl} .00, .04], \mathrm{CFI}=.98, \mathrm{TLI}=.98, \mathrm{SRMR}=0.06)$ also indicated that the perceived infectability and germ aversion scales can be used together as a unidimensional perceived vulnerability scale. These scales had acceptable reliability in the present study (Table 1).

[Please, insert Table 1 about here]

\section{Statistical analysis}

Analyses of the factor structure of the FCV-19S. As the FCV-19s has previously been validated in several contexts (at least 11 languages), a CFA was performed to verify the factor structure of the scale2. Data were continuous but not normal. Therefore, the weighted least estimator (WLS) was used to decrease risk of Type I error (Brown, 2015). In the CFA analysis, goodness of fit was evaluated using the chi-square test. 
However, because the $\chi 2$ test is highly affected by sample size (Bentler \& Bonett, 1980), we also considered the relative $\chi 2$ (ratio $\chi 2$ /df), which is less influenced by sample size. The acceptable relative $\chi 2$ range is from less than two (Ullman, 2006) to less than five (Schumacker \& Lomax, 2010). In addition, commonly applied goodness- of-fit indices were also examined: the Comparative Fit Index (CFI; Bentler, 1990), the Tucker-Lewis Index (TLl; Tucker \& Lewis, 1973), and the Root Mean Square Error of Approximation (RMSEA, Steiger, 1998). Good fit was indicated by a non-significant chi-square test, CFI and TLI values $\geq .95$ (for good, $\geq .90$ for acceptable) and RMSEA $\leq 0.06$ (for good, acceptable fit if

$\leq .08$ ) with $95 \%$ confidence interval (Hu \& Bentler, 1999). Analyses of data should not only be based on fit indices. Close inspection of parameter estimates (e.g., factor loadings, cross- loadings) may also reveal relevant information about the model (Morin et al., 2016).

Reliability. Reliability was assessed with Cronbach's alpha using the commonly reported cut- off values of .70 and .80 (Nunnally, 1978). However, in the case of a factor only including a few items, values between .60 and .70 are considered as acceptable (Hair, Black, Babin, \& Anderson, 2014). The $95 \%$ confidence interval for each Cronbach's alpha was also calculated. Convergent and divergent validity. The convergent and divergent validity investigated by correlations with the various validity measures on the whole sample $(n=316)$. Effect sizes were evaluated according to Cohen's standards: correlations of $r=.10$ to .30 were considered as small,

.30 to .50 were considered as medium, and $\geq .50$ were considered as large. A positive correlation was expected between the French FCV-19S total score and the STAI-A and STAI-B, the HADS (anxiety, depression and total) scores, and with germ aversion, perceived infectability and perceived vulnerability to infectious disease. Positive correlations with disgust, shame, sadness, contempt, fear and anger were also expected. Negative correlations with joy and interest were also expected.

Test-retest reliability. Eighty-six participants ( 81 women and 5 men) completed the French FCV-19S again six weeks after first completing it. Their mean age was 31.48 years $(S D=12.02)$. The test-retest reliability was assessed with intra-class coefficient and its $95 \%$ confidence interval.

All analyses were performed using R version 3.6.1 (R Core Team, 2019). Several R packages (psy, psych, mice and lavaan) were used. Statistical tests were considered significant at $p<.05$. Pre-registration, materials, data and scripts are referenced on the Open Science Framework (

/).

\section{Results}

\section{Confirmatory factor analysis}

The one factor model arising from the literature yielded the following indices $\chi 2(14)=79.69, p<.001$; relative $\chi 2=5.69, \mathrm{RMSEA}=.122(95 \% \mathrm{Cl}[.097, .149], \mathrm{CFI}=.726, \mathrm{TLI}=.589, \mathrm{SRMR}=.201$. These results indicate that the goodness of fit was not optimal. In an effort to make the more optimal model for the data, the modification indices (Mls) were considered. Each modification was assessed by a comparison 
between the two models to choose the best. It was found that the inter-item correlations in the final onefactor model (Figure 1) yielded the following indices: $\chi 2(9)=4.46, p=.87$; relative $\chi 2=0.49$, RMSEA $=.000$ (95\% Cl $[.000, .031]), \mathrm{CFI}=1.000, \mathrm{TLI}=1.044, \mathrm{SRMR}=.016$. This one factor model presented satisfactory internal consistency (Cronbach's alpha $=.87,95 \% \mathrm{Cl}=[.85, .89]$ ). Standardized factor loadings indicated that all items appropriately reflected the unidimensional structure.

[Please, insert Figure 1 about here]

\section{Convergent and divergent validity}

The French FCV-19S total score correlated positively with all expected variables, especially with the DESIV subscale of fear (Table 1). The French FCV-19S total score correlated negatively with joy and interest (Table 1). These results indicated a good convergent and divergent validity.

\section{Test-retest reliability}

The mean French FCV-19S total score for the group of the re-test, at the initial testing (t1) and following a six-week interval (t2), are displayed in the Table 2. The intra-class correlation coefficient (ICC)3 revealed good test-retest reliability for between the test and the re-test assessments (ICC=.76, ICC $95 \% \mathrm{Cl}=[.66$; $.84], \mathrm{F}(85,86)=7.38, p<.001)$.

[Please, insert Table 2 about here]

\section{Discussion}

The aim of the present study was to evaluate the psychometric properties of the Fear of COVID-19 Scale among the French population. As in the original study, the French version demonstrates a stable unidimensional structure with robust psychometric properties. In particular, there was (i) strong internal consistency (as demonstrated by the very good Cronbach's alpha), (ii) acceptable construct validity (as demonstrated by the CFA), (iii) confirmed unidimensional structure as demonstrated by the CFA (see also supplementary materials), (iv) good convergent (especially shown by the large and positive correlation with the feeling of fear), ( $v$ ) good divergent validity (as shown by the negative correlation with the feeling of joy), (vi) and good test-retest reliability (as demonstrated by the intra-class coefficient).

As in a previous validation study (Soraci et al., 2020), although we did not include formal diagnoses concerning disorders (e.g., generalized anxiety disorders, depression), the French FCV-19S total score was 
positively correlated with anxiety and depression. This is in line with previous studies highlighting that long period of infectious pandemic can affect individuals' mental health (Duncan et al.2009; Pappas et al.2009). The French FCV-19S total score was also strongly and positively correlated with intense negative feelings including fear, anger, and disgust. These results are of particular interest as a further investigation of the individuals' negative emotional feelings, and deepen the understanding of psychiatric and psychological consequences of a pandemic.

As highlighted in the Introduction, many mental health problems can arise at a distance from the presence of the virus and health measures (Ornell et al., 2020; Rogers et al., 2020). In addition, one or more new acute waves of COVID-19 are feared in France, as in other countries, in the absence of a disappearance of the virus or the availability of a vaccine. For this reason, the availability of a tool such as the FCS-19S appears relevant. It can be used in the screening of the most severe affective disturbances related to the pandemic, and at a distance from the virologic peaks.

This study has some limitations. First, the sample was modestly sized (although sufficient to test the psychometric properties of the French FCV-19S) and comprised a majority of females leading to the impossibility to test gender differences. Further investigations on bigger and more representative genderbalanced samples are needed to replicate preliminary results provided by the present study and the association between the French FCV-19S and age. It also appears relevant to investigate the extent in which "underlying medical conditions associated with a higher risk of death from COVID-19 (e.g., diabetes, hypertension, coronary heart disease, pre-existing respiratory conditions) may experience increased levels of COVID- 19 fear" (Soraci et al., 2020, p.7). In addition, in view of the global phenomenon of the pandemic, and the international dissemination of this assessment tool, an analysis of population invariance would appear relevant to the cross-cultural stability of the scale.

In conclusion, the French FCV-19S demonstrated good and robust psychometric properties to assess the unidimensional construct of the fear of COVID-19 among French- speaking participants. In short, the French FCV-19S is a reliable, stable and valid tool for assessing the severity of the fear of COVID-19. This scale can be used to better investigate the relationship between emotions and psychiatric and psychological disorders during the COVID- 19 pandemic.

\section{References}

Ahorsu, D. K., Lin, C.-Y., Imani, V., Saffari, M., Griffiths, M. D., \& Pakpour, A. H. (2020). The Fear of COVID-19 Scale: Development and initial validation. International Journal of Mental Health and Addiction. Advance online publication. https://doi.org/10.1007/s11469-020-00270-8

Barbisch, D., Koenig, K. L., \& Shih, F. Y. (2015). Is there a case for quarantine? Perspectives from SARS to Ebola. Disaster Medicine and Public Health Preparedness, 9(5), 547-553.

https://doi.org/10.1017/dmp.2015.38 
Bentler, P. M. (1990). Comparative fit indexes in structural models. Psychological Bulletin, 107(2), 238246. https://doi.org/10.1037//0033-2909.107.2.238

Bentler, P. M., \& Bonett, D. G. (1980). Significance tests and goodness of fit in the analysis of covariance structures. Psychological Bulletin, 88(3), 588-606. https://doi.org/10.1037/0033- 2909.88.3.588

Brooks, S. K., Webster, R. K., Smith, L. E., Woodland, L., Wessely, S., Greenberg, N., \& Rubin, G. J. (2020). The psychological impact of quarantine and how to reduce it: Rapid review of the evidence. The Lancet, 395(10227), 912-920. https://doi.org/10.1016/S0140-6736(20)30460-8

Carbone, S. R. (2020). Flattening the curve of mental ill-health: The importance of primary prevention in managing the mental health impacts of COVID-19. Mental Health \& Prevention, 19, 200185. https://doi.org/10.1016/j.mhp.2020.200185

CDC Centers for Disease Control and Prevention. (2020). Coronavirus Disease 2019 (COVID-19). Centers for Disease Control and Prevention. https://www.cdc.gov/coronavirus/2019-ncov/daily- lifecoping/managing-stress-anxiety.html

Crede, M., \& Harms, P. (2019). Questionable research practices when using confirmatory factor analysis. Journal of Managerial Psychology, 34(1), 18-30. https://doi.org/10.1108/JMP-06-2018- 0272

Duncan, L. A., Schaller, M., \& Park, J. H. (2009). Perceived vulnerability to disease: Development and validation of a 15-item self-report instrument. Personality and Individual Differences, 47(6), 541- 546. https://doi.org/10.1016/j.paid.2009.05.001

Falissard, B. (2011). Analysis of Questionnaire Data with R. CRC Press.

Griffiths, M.D., Mamun, M.A. (2020). COVID-19 suicidal behavior among couples and suicide pacts: Case study evidence from press reports. Psychiatry Research, 289, 113105.

https://doi.org/10.1016/j.psychres.2020.113105.

Guillemin, F., Bombardier, C., \& Beaton, D. (1993). Cross-cultural adaptation of health-related quality of life measures: Literature review and proposed guidelines. Journal of Clinical Epidemiology, 46(12), 14171432. https://doi.org/10.1016/0895-4356(93)90142-n

Gunnell, D., Appleby, L., Arensman, E., Hawton, K., John, A., Kapur, N., Khan, M., O’Connor, R. C.,

Pirkis, J., Appleby, L., Arensman, E., Caine, E. D., Chan, L. F., Chang, S.-S., Chen, Y.-Y., Christensen, H., Dandona, R., Eddleston, M., Erlangsen, A., ... Yip, P. S. (2020). Suicide risk and prevention during the COVID-19 pandemic. The Lancet Psychiatry, Advance online publication. https://doi.org/10.1016/S22150366(20)30171-1

Horn, J. L. (1965). A rationale and test for the number of factors in factor analysis. Psychometrika, 30, 179-185. 
Hu, L. T., \& Bentler, P. M. (1999). Cutoff criteria for fit indexes in covariance structure analysis: Conventional criteria versus new alternatives. Structural Equation Modeling, 6(1), 1-55.

https://doi.org/10.1080/10705519909540118

Izard, C. E., Libero, D. Z., Putnam, P., \& Haynes, O. M. (1993). Stability of emotion experiences and their relations to traits of personality. Journal of Personality and Social Psychology, 64(5), 847- 860. https://doi.org/10.1037/0022-3514.64.5.847

Lin, C.-Y. (2020). Social reaction toward the 2019 novel coronavirus (COVID-19). Social Health and Behavior, 3(1), 1. https://doi.org/10.4103/SHB.SHB_11_20

Mamun, M.A., Griffiths, M.D. (2020). First COVID-19 suicide case in Bangladesh due to fear of COVID-19 and xenophobia: possible suicide prevention strategies. Asian Journal of Psychiatry, 51, e102073. https://doi.org/10.1016/j.ajp.2020.102073.

Ornell, F., Schuch, J. B., Sordi, A. O., Kessler, F. H. P., Ornell, F., Schuch, J. B., Sordi, A. O., \& Kessler, F. H. P. (2020). "Pandemic fear" and COVID-19: Mental health burden and strategies. Brazilian Journal of Psychiatry, 42(3), 232-235. https://doi.org/10.1590/1516-4446-2020-0008

Rogers, J. P., Chesney, E., Oliver, D., Pollak, T. A., McGuire, P., Fusar-Poli, P., Zandi, M. S., Lewis, G., \& David, A. S. (2020). Psychiatric and neuropsychiatric presentations associated with severe coronavirus infections: A systematic review and meta-analysis with comparison to the COVID-19 pandemic. The Lancet Psychiatry, 7(7), 611-627. https://doi.org/10.1016/S2215-0366(20)30203-0 Schmitt, T. A. (2011). Current methodological considerations in exploratory and confirmatory factor analysis. Journal of Psychoeducational Assessment, 29(4), 304-321.

https://doi.org/10.1177/0734282911406653

Schumacker, R. E., \& Lomax, R. G. (2010). A beginner's Guide to structural equation Modeling (2 ${ }^{\text {nd }}$ edition). Taylor \& Francis.

Soraci, P., Ferrari, A., Abbiati, F. A., Del Fante, E., De Pace, R., Urso, A., \& Griffiths, M. D. (2020). Validation and psychometric evaluation of the Italian Version of the Fear of COVID-19 Scale. International Journal of Mental Health and Addiction. Advance online publication. https://doi.org/10.1007/s11469-020-00277-1

Spielberger, C. D., Gorsuch, R. L., Lushene, R., Vagg, P. R., \& Jacobs, G. A. (1983). Manual for the State-Trait Anxiety Inventory. Consulting Psychologists Press.

Steiger, J. H. (1998). A note on multiple sample extensions of the RMSEA fit index. Structural Equation Modeling, 5(4), 411-419. https://doi.org/10.1080/10705519809540115

Tucker, L. R., \& Lewis, C. (1973). A reliability coefficient for maximum likelihood factor analysis.

Psychometrika, 38(1), 1-10. https://doi.org/10.1007/BF02291170 
Ullman, J. B. (2006). Structural equation modeling: Reviewing the basics and moving forward. Journal of Personality Assessment, 87(1), 35-50. https://doi.org/10.1207/s15327752jpa8701_03

Williams, B., Onsman, A., \& Brown, T. (2010). Exploratory factor analysis: A five step guide for novices. Journal of Emergency Primary Health Care, 8(3), 1-13.

World Health Organization (2020). Mental health and COVID-19. Retrieved July 13, 2020, from: https://www.who.int/teams/mental-health-and-substance-use/covid-19

Worldometer. (2020). Coronavirus Update (Live) : 9,392,883 Cases and 480,563 Deaths from COVID- 19 Virus Pandemic. https://www.worldometers.info/coronavirus/

Zigmond, A. S., \& Snaith, R. P. (1983). The Hospital Anxiety and Depression Scale. Acta Psychiatrica Scandinavica, 67(6), 361-370.

\section{Footnotes}

1 Positive emotions refer to joy and interest whereas negative emotions refer to anger, disgust, contempt (Cronbach's alpha $=.60$, Cronbach's alpha 95\% Cl=[.52, .67]), fear, guilt (Cronbach's alpha $=.75$, Cronbach's alpha $95 \% \mathrm{Cl}=[.7, .79]$ ), shame (Cronbach's alpha=.75, Cronbach's alpha 95\% $\mathrm{Cl}=[.7, .79])$, shyness (Cronbach's alpha $=.87$, Cronbach's alpha $95 \% \mathrm{Cl}=[.84, .89]$ ), sadness, and self-hostility (Cronbach's alpha=.82, Cronbach's alpha $95 \% \mathrm{Cl}=[.78, .85])$. In the table we displayed only internal consistency for factors of interest

2 As pre-registered on Open Science Framework priori to theses analyses, both exploratory and confirmatory factorial analyses have been done (see supplementary materials).

3 The ICC was calculating according to the following parameters: total score $(k=86)$, consistency, oneway model.

\section{Tables}

Please see the supplementary files section to view the tables.

\section{Figures}




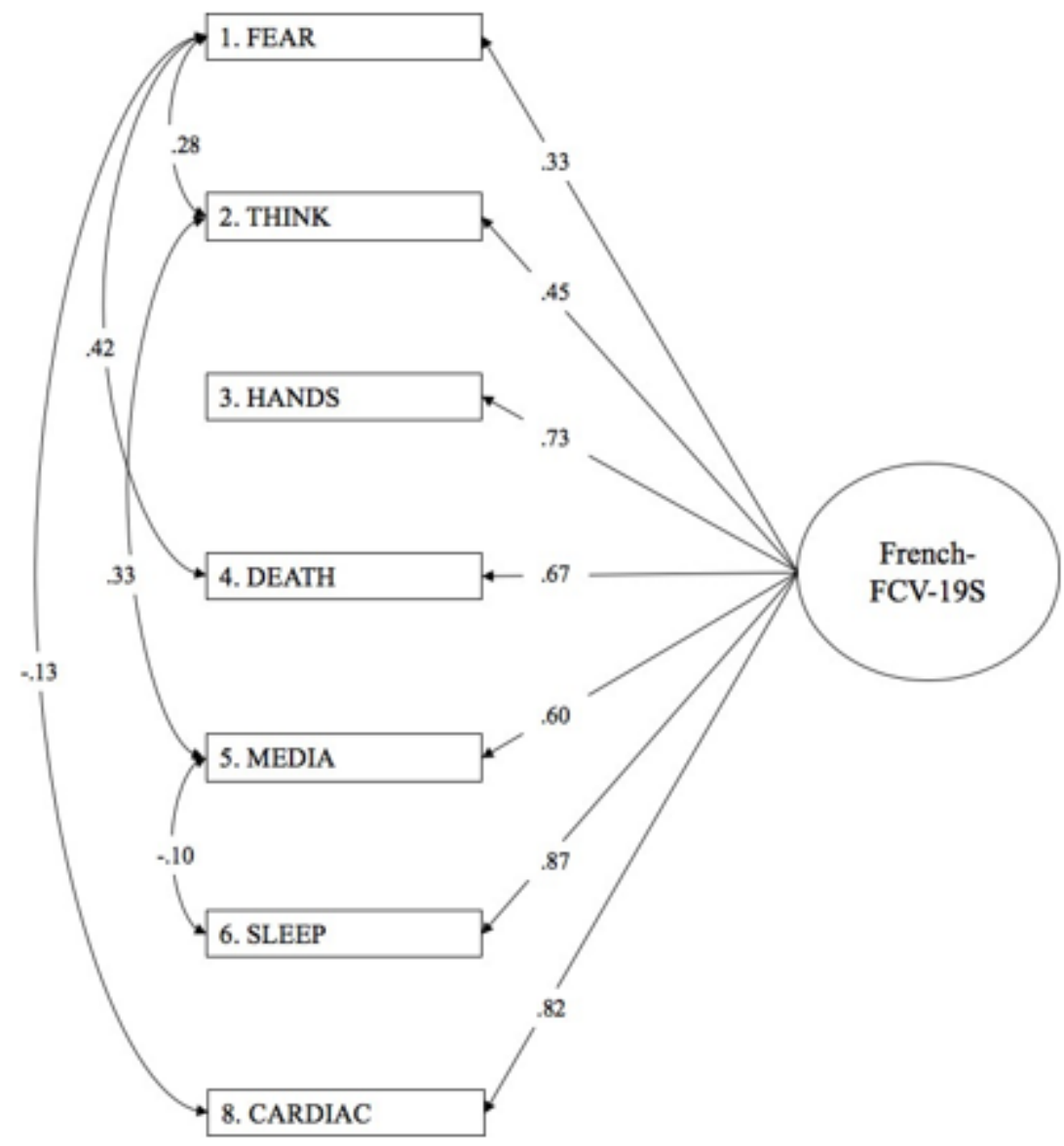

Figure 1

Factorial structure of the French version of the Fear of Covid-19 Scale.

\section{Supplementary Files}

This is a list of supplementary files associated with this preprint. Click to download.

- Tables.docx

- AppendixA.docx 